\title{
Influence of hydrodynamic interactions on the adsorption process of large particles.
}

\author{
I. Pagonabarraga and J.M. Rubí \\ Departament de Física Fonamental, Facultat de Física \\ Universitat de Barcelona \\ Diagonal 647, 08028 Barcelona, Spain
}

(November 16, 2017)

\begin{abstract}
We have studied the adsorption process of non-Brownian particles on a line. As a new ingredient with respect to previously proposed models, we have incorporated hydrodynamic interactions between the incoming particles and the preadsorbed particles as well as the surface. We then numerically analyze the effect of these interactions on typical relevant quantities related to the adsorption process. Comparing our model to the ballistic deposition model (BM) shows in particular a significant discrepancy in the pair correlation function. The results obtained can explain some differences observed between recent experiments and $\mathrm{BM}$ predictions, as reported in ref. [1]. Finally, the limitations of the applicability of BM in experimental situations is addressed. PACS numbers:68.45.Da,81.15.-z,47.15.Gf
\end{abstract}


The adsorption of large particles on solid surfaces is a problem which has been subject to a great deal of interest during the last years. This process is mainly constituted by two basic steps: the transport of the objects from the bulk towards the surface, and their subsequent adhesion to it. A number of models have been proposed in recent years in an attempt to describe the process. In particular, the random sequential adsorption model (RSA) was initially introduced as a simple model which captures the essentials of its kinetics [2]. In RSA the transport of particles to the surface is not considered. Thus, a particle arriving at the surface is accepted if it does not overlap with a previously adsorbed one; otherwise it is rejected. Specific quantities, such as the maximum coverage of the surface, or jamming limit, are in accordance with some experimental results [3] [4] [5], so it was concluded that RSA was a good model when particles diffuse to the surface. However, further numerical studies which took into account the diffusion showed discrepancies in the pair distribution function [6]. To check the validity of RSA, experiments on the deposition of colloidal particles have been performed [1]. In these experiments, gravity has been shown to play an important role, and therefore it seems more appropriate to compare with the results predicted by the ballistic model (BM) [7]. In this model, particles approach the surface following straight trajectories, and are accepted if no preadsorbed particle is present or if there is room at its adjacent region. In the latter case, the particles roll down the surface of the preadsorbed ones [8]. If there is no room, the particle is rejected. Comparison of the pair distribution function observed experimentally with BM results exhibits some discrepancies, which may, in principle, be attributed to polydespersity, van der Waals forces (vdW) or hydrodynamic interactions (HI). Preliminary results [9], however, have shown that polydispersity does not explain this disagreement.

Our purpose in this Letter is to address the effects of HI in the adsorption process. Up to now, HI have only been considered in a situation in which gravity can be neglected [10]. In this case, as the particle diffuses more easily paralelly rather than perpendicularly to the plane due to HI, no strong differences are observed with RSA predictions. However, if gravity is present, the randomization effect of the diffusion dissapears, and, therefore, no 
guess can be made about the importance of HI on the adsorption kinetics.

To elucidate the effects of HI we have proposed a one-dimensional model in which spherical particles of radius $a$ falling down one by one towards the adsorbing line due to the presence of a gravity field, interact with the nearest preadsorbed particles and with the surface. When they reach the line they are accepted on the surface if there is enough room for them; otherwise, they are rejected. Once the particles are adsorbed, they stick on the surface. In this respect, the kinetics is quite similar to the one prescribed by BM, although the rolling now can be performed without touching the adsorbed sphere (see Fig. 1a). Moreover, during the process the incoming particle is assumed to interact only with its nearest neighbors on the line. We have analysed our system under the conditions such that inertial and diffusion effects may be neglected. Finally, in the treatment of HI we have assumed additivity of the friction tensors. This standard approximation, already introduced in Ref. [11] [10], considerably simplifies our treatment, and it is sufficient to make evident the effects of $\mathrm{HI}$ in the adsorption process.

Under these approximations, the dynamics of the incoming particle is governed by the equation

$$
\vec{v}=\overrightarrow{\vec{\mu}} \cdot \vec{F} \equiv m_{\text {app }} \overrightarrow{\vec{\mu}} \cdot \vec{g}
$$

Here $\vec{v}$ is the velocity of the particle, $\overrightarrow{\vec{\mu}}$ its mobility tensor, $\vec{g}$ the acceleration of gravity, and $m_{a p p}=4 \pi a^{3} \Delta \rho / 3$ the apparent mass, with $\Delta \rho \equiv\left(\rho_{p}-\rho_{f}\right), \rho_{p}$ being the density of the particle and $\rho_{f}$ the density of the fluid. Note that in eq.(11) we have not considered van der Waals forces with the plane since, contrary to what happens for Brownian particles [10], they are not relevant in the dynamics of large particles due to its short range nature. The expression of the mobility matrix takes into account the existence of HI. Under the additivity approximation, the friction tensor $\overrightarrow{\vec{\xi}}$, which is the inverse of the mobility matrix, splits up into contributions due to the surface and to the already attached spheres. As only nearest neighbor interactions are assumed, this last contribution is reduced to the contributions coming from the presence of the two nearest adsorbed spheres. One has 


$$
\overrightarrow{\vec{\xi}}=\overrightarrow{\vec{\xi}}_{s p}+\overrightarrow{\vec{\xi}}_{s 1}+\overrightarrow{\vec{\xi}}_{s 2}-2 \xi \overrightarrow{\overrightarrow{1}}
$$

where $\overrightarrow{\vec{\xi}}_{s p}$ is the friction tensor of one sphere in the presence of a plane, without any other sphere being present, and $\overrightarrow{\vec{\xi}}_{s 1}$ and $\overrightarrow{\vec{\xi}}_{s 2}$ are the friction tensors of two isolated spheres; the indexes 1 and 2 referring to the two nearest neighbors. In the limit when the particle is far from the line, the friction tensor tends to the well-known expression $\xi \overrightarrow{\overrightarrow{1}}$, with $\overrightarrow{\overrightarrow{1}}$ being the unit matrix and $\xi=6 \pi \eta a$ with $\eta$ the viscosity of the fluid. Therefore, both the tensorial character and the spatial dependence of the friction are due to the presence of other objects. In particular, due to stick boundary conditions, the friction tensor diverges when the particle touches any solid surface, the divergence being different depending on the direction of movement. Lubrication forces then hinder any contact between objects. Explicit analytic expressions for these tensors, which depend on the distance between the particle and the line and on the relative position of the particles, have been given by Brenner [12 for the case of sphere-plane friction (eqs.(2.19) and eqs.(2.63 and 2.65a) respectively) and by Jeffrey-Onishi (eqs. 3.20,4.19,5.9 and 7.14 ) [13] for the sphere-sphere friction tensor. It should be noted that, although particles are forced to adsorb on a line, the expressions used for the friction tensors are three-dimensional, as we have real three-dimensional adsorbing spheres.

In order to gain a physical understanding of the results obtained in the adsorption kinetics, it is interesting to study the effect of an adsorbed particle on the dynamics of another one coming from the bulk. To this end, we have numerically solved eq.(1). In the region where the particles are close together, the mobility decays rapidly to zero. Therefore, the numerical algorithm should contain a variable time step in order to ensure that the mobility element associated with the perpendicular motion of the particles does not change significantly during one integration step. The unit of length has been taken as the diameter of the spheres, and the unit of time $9 \nu \rho_{f} /(a g \Delta \rho)$, with $\nu$ the kinematical viscosity of the fluid. In these units, eq.(11) is dimensionless and, therefore, does not depend on either the kind of particles or on the medium. This means, that the final position of the incoming particles will not depend on their mass or volume. The initial conditions are such that the incoming 
particle is at a heigth of 50, at which HI are negligible, and the horizontal position starts close to one of the adsorbed particles and is progressively displaced from the axis. In this way, we have studied the final position of the incoming sphere, $x_{f}$, as a function of its initial distance to the axis, $x_{d}$ (see Fig. 1a). Due to the divergence of the friction tensor when the particles are in contact, the calculation stops when the sphere is almost touching the plane. vdW are effectively taken into account by stopping the simulation close to the plane. When $d$ is large enough, the results are plotted in Fig. 1b where we compare our results with the predictions of BM, and from which we can infer the modifications introduced by HI. In the vicinity of the attached particle, these corrections are to the order of $10-15 \%$ with respect to BM. They originate from the fact that HI cause an increase of the friction coefficient of the particle, which depends on its relative motion with respect to the adsorbed one. Thus, whereas the friction coefficient for the perpendicular motion of two close objects at a distance $r \equiv s+2 a$ diverges as $1 / s$, the corresponding coefficients for parallel motion diverge as $\ln s$. The result is an effective repulsion between the particles. We have found that the final position predicted by HI differs from the one given by BM up to values $x_{d} \sim 5$. Thus, the effects of HI persist far from the attached particle. For finite $d$ the discrepancies with respect to $\mathrm{BM}$ are restricted to a closer region in the vicinity of the attached particle but are essentially of the same order of magnitude. This effective repulsion will be responsible for the differences observed in the adsorption process.

We have numerically studied the deposition of spheres on a line. For this purpose, we have considered a line of length 800. At a height of 50, positions are chosen randomly. Once one position has been selected, it is taken as the initial condition for the sphere, and subsequently eq.(11) is solved numerically until the sphere reaches the line. In order to speed up the program, if the incoming particle nearly touches a preadsorbed one, and due to the results shown in Fig. 1b., it is accepted in the line according to BM rules. If room is available, the particle is adsorbed, otherwise it is rejected. Then, another position is randomly selected, and the process goes on until either a prescribed fraction of covered line, $\theta$, or the jamming configuration $\theta_{\infty}$, in which there is no more room available for incoming 
particles, is reached. Using periodic boundary conditions, we then generate configurations of adsorbed particles from which we can study the relevant quantities related to this process.

In this sense, we have analyzed the radial distribution function, $g(r)$, of the adsorbed particles at different concentrations. In order to obtain representative values of $g(r)$, approximately 1500 realizations of the adsorption process have been performed. In Fig. 2a the $g(r)$ at coverages $\theta=0.25, \theta=0.5$ and at the jamming limit are displayed. As expected, $g(r)$ decays faster and the height of the peaks increases when increasing the coverage, as in the BM case, although the initial decay after the peak does not depend too much on it. The differences between the $g(r)$ corresponding to their coverages are not significant at distances larger than 3. Furthermore, in Fig. 2b we have compared $g(r)$ with the one given by BM. We have observed a smooth decay of the function behind the peak in contrast with the sharp decay predicted by the BM model. In the region of the first peak large differences are observed, for example, at $r=1.3$ it is of order $12 \%$, and of $42 \%$ at $r=1.2$. The $g(r)$ when HI are considered tends smoothly towards the corresponding quantity predicted by $\mathrm{BM}$ and at $r=1.5$ the difference is $6 \%$. These differences tend to decrease when increasing the coverage, although even at jamming some diferences are observed.

To investigate the effect of $\mathrm{HI}$ on global quantities, from our model we have computed the available fraction of line as a function of the coverage. Figure 3 shows that differences, which are always smaller than $1-2 \%$, are less significant than the ones obtained for the radial distribution function. Moreover, it has no relevant effect on the time evolution of the coverage either, as shown in the insert of Fig. 3, whose assymptotic temporal behavior is equal to the one prescribed by BM. At intermediate times the coverage deviates from BM predictions, leading to a jamming limit $\theta_{\infty}=.797$, slightly smaller than the BM one, $\theta_{\infty}^{B M}=.810$

In summary, we have shown that in respect to macroscopic quantities of the deposition process, our model introduces no important corrections to BM results. The explanation of this result lies in the fact that these quantities can be considered as averages over the line, and therefore local details are masked. However, local properties are strongly affected by 
HI. In particular, the distribution of spheres around a preadsorbed one at low coverages shows differences up to $10 \%$ due to the effective repulsion induced by HI. In regards to the radial distribution function, the decay after the peaks is slower than in BM. Behind the first peak, differences can be as large as $40 \%$, and the convergence towards BM is slow. Discrepancies after the second peak are also observed, though they are smaller. This behavior decreases slightly with the coverage, so that even at jamming some differences are observed. Our conclusion is that HI cannot be neglected a priori when studying such properties. Recent experimental results for the adsorption of colloidal particles on a surface [1] show that the radial distribution function deviates from BM predictions, which is the same kind of behavior shown in Fig. 3. Though our simulations have been perfomed in $1 d$, they capture the essential features of the process, and explain the differences between experiments and $\mathrm{BM}$ predictions, which do not originate from the polydispersity of the solution [9]. In $2 \mathrm{~d}$ these differences can be expected to be smaller than the ones reported in this Letter because of the additional angular average performed to arrive at the radial distribution function. BM has been introduced as the limiting case when the deposition is controlled by gravity instead of diffusion [7]. However, we have shown that in the regime when gravitational effects become important, $\mathrm{HI}$ introduce significant effects. BM could then reasonably describe the physics of the deposition process only when inertial effects become dominant, since in this regime the damping term is negligible. This fact occurs for times not larger than the inertial time $\tau \equiv m / \xi=2 a^{2} \Delta \rho /\left(9 \nu \rho_{f}\right)$. In usual experimental situations, as the one reported in ref. 四, $\tau \sim 10^{-6} s$, while the experimental time scale is of the order of minutes. Consequently, in this situation inertial effects are negligible, which explains the disagreement between the radial distribution function obtained experimentally and the one calculated from BM, and justifies the validity of eq.(四). Therefore, the applicabilitty of BM is severely restricted in experimental situations. Finally, the fact that HI affect the local distribution of adsorbed particles implies that these interactions will be relevant when studying other physical properties of adsorbed layers, as for example the dielectric susceptibility of adsorbed particles [14]. 


\section{ACKNOWLEDGMENTS}

We would like to acknowledge Profs. P. Schaaf and D. Bedeaux for fruitful discussions. This work has been supported by the European Economic Community under grant SCI*CT91-0696 and by CICYT (Spain), grant PB92-0895. 


\section{REFERENCES}

[1] P. Wojtaszcyk, P. Schaaf, B. Senger, M. Zembala and J. C. Voegel, J. Chem. Phys 99, 7198 (1993).

[2] E. L. Hinrichsen, J. Feder, and T. Jossang, J. Stat. Phys. 44,793 (1992).

[3] J. Feder, J. Theor. Biol. 87, 237 (1980); G. Y. Onoda and E. G. Liniger, Phys. Rev. A 33, 715 (1986).

[4] J.J. Ramsdem, Phys. Rev. Lett. 71, 295 (1993).

[5] Z. Adamczyck, B. Siwek, M. Zembala and P. Weronski, Langmuir 8,2605 (1992).

[6] B. Senger, J.-C. Voegel, P. Schaaf, A. Johner, A. Schmidt and J. Talbot, Phys. Rev. A 44, 6926 (1991).

[7] J. Talbot and S. Ricci, Phys. Rev. Lett 68, 958 (1992).

[8] P. Meakin and R. Jullien, J. Phys. A 25, L189 (1992); A.P. Thomposon and E.D. Glandt, Phys. Rev. A 46, 4639 (1992); P. Viot, G. Tarjus and J. Talbot, Phys. Rev. E 48, $480(1993)$.

[9] P. Wojtaszcyk and P. Schaaf, private communication.

[10] J. Bafaluy, B. Senger, J.-C. Voegel and P. Schaaf, Phys. Rev. Lett. 70, 623 (1993).

[11] G. Bossis and J.F. Brady, J. Chem. Phys. 80, 5141 (1984).

[12] H. Brenner, Chem. Eng. Sci. 16, 242 (1961), A.J. Goldmann, R.G. Cox, and H. Brenner, Chem. Eng. Sci. 22, 637 (1967).

[13] D. J. Jeffrey and Y. Onishi, J. Fluid Mech. 139, 261 (1984).

[14] M.T. Haarmans and D. Bedeaux, Thin Solid Films 224, 117 (1993). 


\section{FIGURES}

FIG. 1. a) Illustration of the geometry of the model. An incoming particle is selected at a height $h_{0}$ at a certain distance $x_{d}$ from the nearest particle. A second nearest neighbor is located at a distance $d$. The particle will end in the position $x_{f}$. b) Final position as a function of the initial displacement when $d$ is large enough. Insert, the difference in the final position predicted by $\mathrm{HI}$ and $\mathrm{BM}$ relative to the one given by BM.

FIG. 2. a) $\mathrm{g}(\mathrm{r})$ for the HI model at coverages $\theta=0.25,0.5$ and jamming. b) Comparison with BM at $\theta=0.5$
FIG. 3.

curves are practically indistinguishable up to high coverages. Insert, the coverage as a function of time for BM and $\mathrm{HI}$. 